\title{
The 'study walk-through': a method to help translate your protocol into an accurate and successful study
}

\section{Trudie Lang ( $\nabla$ trudie.lang@ndm.ox.ac.uk)}

University of Oxford https://orcid.org/0000-0003-2273-5975

\section{Research Article}

Keywords: Clinical operations, clinical studies, Ebola virus, malaria, methods, podoconiosis, pragmatic trial, study walk-through, vaccines

Posted Date: March 1st, 2022

DOI: https://doi.org/10.21203/rs.3.rs-1145790/v1

License: (c) (1) This work is licensed under a Creative Commons Attribution 4.0 International License. Read Full License 


\section{Abstract \\ Background}

The operational planning and delivery of a clinical study determines whether it can answer the question that it has set. Strong methodology is needed to ensure that the study endpoints are measured accurately, safely and ethically. The study walk-through is a method to ensure that every step in a study protocol is carefully considered in its operational context, and that those responsible for undertaking the step are tasked with overseeing it appropriately. This article describes the study walk-through approach, with the aim of informing others about its use.

\section{Methods}

To implement the study walk-through method, the entire study team is gathered as early as possible in the planning of the study. An overview of the study protocol is presented, focusing on the primary endpoints and how these will be measured. One team member then leads the process of describing the study step-by-step, as it would be operated at the study centres. Each team member is encouraged to imagine each step from the participant's perspective. The process covers steps from community engagement and recruitment, through each visit, until the end of the study. The processes and data collected are discussed in the context of actions that relate specifically to the participants, e.g. clinical assessments. The coordinator leads the discussion of each study step, which is recorded as the discussion flows.

\section{Results}

The study walk-through method has been applied and refined in highly varied clinical studies over 20 years. Across the three examples presented, which span malaria, podoconiosis and Ebola virus research, the method was used to anticipate and address a diverse range of operational issues. These included issues relating to protocol design, the recruitment and retention of study participants, randomisation, maintaining study blinding, primary endpoint measurement, transporting samples, data entry and followup.

\section{Conclusions}

This effective method ensures that each team member fully understands the protocol and research question, allowing them to identify elements of their role that are key to the study outcome. This process also uses the experience of each team member to inform the design and operational planning of the study, thereby raising standards and reducing errors. 


\section{Background}

There is a need for evidence from all types of clinical research: from observational, sampling-only studies through to clinical trials. However, the failure of clinical studies and the publication of inaccurate or misleading data can compromise this evidence [1]. Clinical studies can fail or lose validity for various reasons, including operational issues such as poor recruitment [2] or loss to follow-up [3, 4]. Inaccuracies sometimes occur within studies because of error or imprecision in the process of defining or measuring a parameter, or because of missing data $[4,5]$. To enable research teams to run studies that can reliably answer the question that they have set, we need to consider operational methodology as being at least as important as the statistical design within the protocol.

The process of turning a question into a protocol and a protocol into an operational study is an overlooked area, and undervalued in terms of being a fundamental methodology area in clinical research. A study can utilise the most impressive, cutting-edge diagnostic technology or new statistical approach, but if the key endpoints have not been considered carefully, the study is difficult to implement in the clinical setting, or the study team are not conducting the processes in an accurate way, then errors can occur. Such errors may even go unnoticed.

Study protocols and standard operating procedures (SOPs) are commonly written away from the study site and without the involvement of the study team. As a result, generic SOPs can be produced that might set a standard and attempt to ensure that multiple centres run all their processes in the same way, but are unpractical, inappropriate and do not reflect the real situation in the clinic or laboratory. The critical steps in a process, during which something could go wrong, may not be recognised or covered by a generic SOP and therefore, could be managed differently by different staff, incorrectly or not managed at all during the whole study period. All study staff involved, including the study monitor, need to understand the relative importance of these critical steps to the final study findings. For this reason, it is important that the whole study team fully understands the protocol and the research question it is addressing. Mistakes and inaccuracies can go unnoticed in clinical studies, but informed team members who understand the science spot issues that others may not have thought of.

'The study walk-through' is an approach that was developed and refined in Kenya. At the time of its development, there were many studies being run, ranging from complicated, regulatory standard vaccine trials in the Kenyan community through to paediatric trials in high dependency units. It was clear that an approach was needed to make sure that the studies ran well in the challenging clinical settings in which they were to be conducted.

Taking the whole team through a study walk-through process allows everyone to foresee the whole study as it rolls out, from the participants' experience through to what happens to their samples and their data. This process has always flagged potential issues that we had not considered when writing the protocol. Unless you consider every step from the perspective of the team member tasked with delivering that step, it is not possible to consider everything, because you cannot know the setting and the constraints as they do. SOPs written from afar fail precisely because of this. 
Based on over twenty years of turning protocols into operational studies, it is clear that studies perform much better if the entire team are engaged from the earliest possible point. Every team member plays an important role in the study, and their understanding of the study, what to measure, and why taking personal responsibility for data accuracy is both important for the patient in front of them, and also for potentially thousands of others in the future who may (or may not) receive a new treatment based on the outcome of this study, is fundamental to its success. For example, when they are writing ' 4.2 ' in a patient's notes and then entering this value into the case report form (CRF) or database, they understand where 4.2 came from and why it is so critical for the study that 4.2 is really 4.2 and not 42 or 4.0 . Here, 4.2 is a theoretical example that could represent a $4.2 \times 10^{12} / \mathrm{L}$ red blood cell count in a malaria study, in

which change in red blood cell count is a key endpoint. If team members understand that their role in ' 4.2 ' being measured and recorded accurately matters to the final answer that the study delivers, then whether they are the study nurse drawing the blood, or the laboratory technician receiving the sample, or the study coordinator transcribing the laboratory data, they feel much more ownership of the task and part of the endeavour to answer this important, unaddressed question.

The objective of the study walk-through is to identify and mitigate against the occurrence of errors that could jeopardise the accuracy, safety or ethical conduct of studies. It is a practical and achievable way to make sure that every step in a study protocol is carefully considered in its operational context, and that those responsible for undertaking the step are tasked with overseeing it appropriately. This article describes the study walk-through approach and three examples of its application, with the aim of informing others about its use.

\section{Methods}

\section{Overview}

The study walk-through method aims to identify every situation in which a consequential error could occur, and mitigate against this using a SOP, through training, or by changing a process.

To implement the method, the entire study team is gathered as early as possible in the development of the protocol. Ideally, this meeting would take place long before the protocol is finalised and submitted for regulatory approval, as this can reduce the need for protocol modifications and amendments later on. At the beginning of the meeting, an overview of the study protocol is presented. This overview focuses on how the participants move through the study visits and interventions, and then on the primary endpoints and how these will be measured. If everyone on the team understands what needs to happen when and what matters in terms of capturing the key outcomes, then a successful study can be set up and a highly operational protocol can be generated.

The study is then 'walked through'. We find that this works best when a large piece of paper, coloured pens and a big table are used at the meeting. One team member, such as the study coordinator, leads the process of describing the study step-by-step, as it would be operated at the study centres. Each team 
member is encouraged to imagine the experience of the participant, from when and where they first learn about the study, to them being enrolled, then receiving the intervention, and then attending the follow-up visits, the collection of the participant's samples and the information that this will provide. The coordinator leads the discussion of each study step, asking the team to consider who will be responsible for each step, where and when each step will happen, and how all steps could be carried out to ensure: the best experience for the participants and their families; the best experience for the study and healthcare staff involved; and adherence to the protocol. The outcomes of the discussions are recorded as the discussion flows. Once the 'journey' of a participant through the study visits has been considered by the team in this way it becomes clear where SOPs will be needed. This study walk-through method provides a detailed view of the whole process and thereby highlights where things could go wrong.

Potential topics for discussion at the study walk-through meeting are summarised in Figure 1. The discussion would broadly follow the following steps, depending on the study design and complexity of the protocol.

\section{Screening and enrolment}

\section{Approaching potential participants}

The team would discuss how potential participants would be approached and what the requirements might be for community engagement [6-9]. They may consider questions such as: is there a community advisory board in place? Is this a study of a chronic condition for which there are lists of patients who could be approached? Or is this a study of an infectious disease in which you will need to wait for patients to present with the condition? Where are these patients seen? If it is in the community, then what permissions will be needed? How can the community be consulted and involved? What clinic or health facility is this going to happen in? Will study staff or other staff be working for the health facility? Would it be important to brief the other staff members? Are there adverts being produced for this study? How can recruitment be optimised?

Key factors to consider with respect to screening and enrolment are how long it will take and how many people it will need. If the study has a screening step, the team should discuss how long it will take and what it will involve. This will give an idea as to what staffing levels, facilities and training will be needed, and an idea of capacity. How many people could be recruited into the study in one day, for example? Might this depend on clinic rooms, beds, staffing or laboratory capacity? Discussing this with the people who will conduct the study, in their working environments, will answer such questions.

Additional questions that may be addressed as part of this walk-through procedure include the following: how are the inclusion and exclusion criteria being applied, and are they going to work? Can reminder cards be made that outline these selection criteria, to be given to clinic staff to remind them about this study and to help identify potential patients? Does this study require laboratory or other diagnostics? Is this step part of standard care or a study procedure? 


\section{Taking consent to participate in the study}

The team would also discuss issues around taking consent to participate in the study. They may consider questions such as: At what point is consent needed? Consent is usually needed before the diagnostic step if it is not normally performed in that patient population $[10,11]$. If patients are being considered for a study, who is going to explain this to them? Will the same person be seeking their consent to participate in the study? Where and when will this happen? How long will it take, and is the timing important? In an emergency setting or when a treatment needs to be administered, slowing down time to treatment would be unethical.

An additional point to consider is what is expected from the participant during the study relative to what would normally happen to a participant with this condition, in this setting. What is being asked of the participant beyond expectations of them during standard care? Does the information sheet and consent form capture this properly?

Other potential problems which could arise with taking consent to participate in the study should also be included in discussion. For example, are the participants vulnerable (children, unconscious, distressed, or unable to read or speak the relevant language)? What needs to be put in place to manage any of these situations? If a witness is needed, what if no-one is available? Is there a viable alternative approach, such as deferred consent [12-14]?

\section{The study visits}

The team would consider requirements for each of the study visits and any practical issues they may present. For example, will the participants progress into the first visit straightaway? This is common for studies of infectious diseases, but less so for studies of vaccinations or chronic conditions. Where would the first visit happen and what is needed? For example, how many participants could be managed at any one time? What would be the constraints? The time that the procedures take? Beds, staffing, laboratory capacity? How many other people may also be attending the study centres for follow-up visits or other clinics? Is this feasible?

The team would also discuss what should happen to the participant during the study visits and during follow-up. For example, is the first visit when baseline samples and information will be taken and/or when the participants will be given an intervention? When will the participants need to return for subsequent visits? Is this beyond what would be normal in their usual care? What could be done to make sure that they do come back for subsequent visits? Or will they be visited at home? What could go wrong here? Would there be any concerns about privacy or stigma that might cause participants to change their minds? How well trained are the staff conducting these visits?

\section{Results}


The study walk-through method has been applied and refined in highly varied clinical studies over 20 years. The practical application of this method is illustrated below, using three examples. For each example, results of using this approach are described.

\section{Example 1: a regulatory vaccine trial}

As one of the centres in the phase III RTS,S/AS01 malaria vaccine trial (NCT00866619) [15-17], we needed to plan how we would set this study up in three distant community health clinics and then bring the samples back to the hospital. We gathered the team, which included the fieldworkers who were based out in the community, the laboratory team from the hospital, the nurses who would be vaccinating the children and all the study coordinators and data managers. We also included a group who were fundamental: the study drivers. Together, we 'walked through' the process of enlisting mothers in the clinics, having them return for the vaccination of their enrolled infants, and following participants up at home. Alongside this, we considered how we would get the samples from the clinics back to the laboratory, and how we would enter the data into the CRFs when the study teams were back in the trial office. Working through these steps made many potential challenges and solutions clear. Some examples of these are described below.

\section{Recruitment and retention of study participants}

We discussed enlisting mothers, recruiting their children and keeping them engaged in the trial. Poor recruitment and retention of children had been identified as the most likely risk to the trial. Therefore, we asked our community engagement team to attend our study walk-through meeting, to advise us how best to deliver the patient information within the specific settings of each clinic. The community engagement team also advised us how to turn consent into an ongoing process, to maintain the trust and support of the families $[6,7,18]$.

\section{Transporting samples}

We planned the study during the dry season, but the drivers pointed out that conditions would be very different during the monsoon. We had a very precise time window to transport samples from the clinics to the laboratory for immunology assays, which were a key outcome in the trial. As a result of our meeting, we were able to switch from cars to motorbikes, so no samples were delayed or lost during the rains.

\section{Data entry}

The volume of information to be gathered from the communities was huge, but there were no formal patient records. Therefore, we needed to create source data sheets for use in the community. We also needed to create a strong system for the data entry clerks, to facilitate taking data from the source data sheets and entering the required information into the CRFs. The study walk-through process led to the setting up of this system and to having one of the data entry team out with the clinical team members every day. This made the whole process better and faster, and reduced the likelihood of errors or missing data. 


\section{Vaccinating and maintaining study blinding}

We needed to blind the participants and the trial team to which vaccine the children were given. As part of the study walk-through process, we worked through the practicalities of applying the protocol and SOP for study blinding, and considered what would work best at each clinic. We identified the solution of using different nurses for the preparation and administration of the vaccines, and carrying these processes out in different rooms.

\section{Measuring the primary endpoint}

Active and passive case detection of malaria episodes determined the primary endpoint of this trial. Involving the fieldworker who would be responsible for this detection in the planning of their own training, and in the planning of the execution of this step, was critical. Training of the fieldworkers was found to be the most critical element, as we needed to ensure consistency throughout the 12 months of follow-up. Therefore, a comprehensive training programme was devised to ensure that quality and standards were maintained throughout the trial, and that the fieldworkers felt fully supported.

\section{Example 2: a pragmatic disease management trial}

This trial (ISRCTN67805210) [19] was conducted because earlier studies had estimated that one million people in Ethiopia were living with podoconiosis [20], one of the most neglected tropical diseases [21]. Pilot studies had suggested that these patients could benefit greatly from foot hygiene support and care $[19,22,23]$, including a highly pragmatic system that involved cleaning and bandaging the affected limbs. However, more supporting evidence was required before additional health providers would administer this treatment. Therefore, a randomised clinical trial was designed. This would be incredibly challenging to conduct, as the trial would need implementing by health workers in an area where there was no previous experience in research. Consequently, during the very early stages of trial initiation (once there was a near-finalised protocol), the team was gathered in the remote area of Ethiopia where this trial was planned. The aim of the team meeting was to allow everyone who was to be involved in the trial to think about how it could work in practice (Figures 2-3). Through this exercise, several key issues were highlighted, as described below.

\section{Randomisation}

The team discussed how to avoid patients sharing their intervention, e.g. in the event that a couple were randomised to the different trial arms. As a result, we decided to switch to a cluster randomisation approach, so that different clinics would use differently timed interventions.

\section{Measuring the primary endpoint}

The primary outcome of the trial was the change in the number painful episodes over 12 months. As highlighted by the study walk-through process, the matter of how to accurately capture the number of painful episodes was a major issue in operationalising this trial. The team discussed how patients could 
report symptoms, and whether patient diaries would work. They considered whether patients would be able to read and write, and if pictures could be used to help report symptoms. The process of working through the draft protocol with community health workers allowed potential difficulties to be identified, and led to the development of simple diaries that the patients used to record these attacks.

\section{Follow-up}

The team discussed how a balance could be struck regarding the number of follow-up visits. The aim would be to see patients often enough to record whether they were experiencing an improvement, without making the study visits so frequent that they would become an intervention in their own right.

\section{Training of study staff}

The study walk-through process identified areas of difficulty that might be encountered during the study, particularly in terms of maintaining the same methods and standards across all visits. It highlighted where training would be needed. As a result, fieldworkers were trained to implement the SOPs, but also to understand why the research was different from standard care and why the protocols were important. The critical nature of the fieldworkers' roles in the success of the trial was emphasised.

\section{Example 3: a trial in a disease outbreak}

During the Ebola virus epidemic, there was the opportunity to conduct the first therapeutic clinical trials during a disease outbreak [24]. Typically, setting up a trial during such an outbreak is impossible because the process takes so long [24]. When planning a phase 2 trial for the treatment of Ebola virus disease (PACTR201411000939962) [25], we undertook the study walk-through process at a very early point, before we knew which drug we would be evaluating. We used this process to set out the draft protocol, pinpoint where SOPs would be needed and identify the trial team's training needs.

The most critical factors for this trial's design and operation were contextual. The trial would include seriously unwell participants, and the trial staff would be working in a very dangerous setting. The trial staff would only be able to see the patients for a very short time. The study walk-through process was invaluable in guiding the protocol development in this situation (Figure 4).

The starting point for the study walk-through discussion was the person with suspected Ebola infection presenting for diagnosis. All we knew at this point was that patients would arrive, be screened in the screening tent, and then admitted to the isolation/treatment tent once they were confirmed to have Ebola virus. There was a high mortality rate among these patients, with around $70 \%$ not surviving past day 7 . Using the study walk-through method, we sketched out the screening and isolation/treatment process and considered the most appropriate efficacy endpoint for evaluating the potential treatments. Using this process allowed us to understand what could practically be measured and when. It was clear that measuring fevers or assessing symptoms was not feasible; therefore, the clear outcome of survival at day 14 was agreed. 
During the study walk-through discussion, it became very clear where significant issues could arise. These issues related to: enrolment within the query period of confirmed diagnosis; dosing and observed dosing; sampling during the follow up-period; and verifying survival. Identifying these major challenges highlighted where SOPs would be needed and guided the design of the trial.

The study walk-through process was undertaken on the day that the trial's funding was secured. At the time, it was not known exactly where the trial would be conducted, or which drugs would be available to evaluate in the trial. However, the final protocol hardly differed from what was set out during these initial discussions. This demonstrates the benefits of considering patients' experiences as they progress through the research process, and thinking about how samples and data will need to be gathered in this context. An important consideration for all studies is whether the research processes might impact on standard clinical care and treatment. Identifying any potential issues in this respect is key to mitigating any conflict between standard care and research.

Overall, the study walk-through process allows a protocol to be written that is highly workable, and that reflects the realities for the participants and study staff in the relevant healthcare settings.

\section{Discussion}

The study walk-through is an operational approach to help ensure that a study can be successfully run in a specific setting. The purpose of this process is to reduce the likelihood of errors occurring that could result in inaccurate data, put the participants at risk, or jeopardise their rights.

Creating a formal process that provides study teams with the opportunity to consider every step required in a study engages them, and can train them on the importance of the study. This process also makes sure that the study protocol and SOPs are designed specifically for the team's setting, and puts the participants and the practical realities of the study site at the centre of operational planning and protocol development.

A limitation of the study walk-through is that it requires some initial time investment from the whole study team. However, the benefits gained from the process clearly outweigh this cost. The study walk-through method has been used in various clinical studies over 20 years, but not each of these studies could be described within the confines of this article.

\section{Conclusions}

The study walk-through process considers a study step-by-step and involves the whole team. This effective method can produce a plan to deliver faster and/or higher quality evidence, whilst engaging everyone involved, well before the start of a study.

Translating a study protocol into what happens in practice is not an administrative function: rather, it needs to be a carefully considered scientific process. Trial methodology is a growing research area which, 
to date, has largely focused on study design and statistics. Surely the process of turning a protocol into a set of steps that take place in clinics, hospitals and laboratories - to answer a scientific question merits more attention from methodology researchers and funders? Those conducting research studies should report how they approached these practical questions. Running methodology studies within other studies is also an important research opportunity that could capture and validate methods and approaches. In this article, the study walk-through approach has been reported and its application described using three examples, with the aim of it guiding others.

\section{Abbreviations}

CRF

case report form

SOP

standard operating procedure

\section{Declarations}

\section{Ethics approval and consent to participate}

Not applicable

\section{Consent for publication}

Not applicable

\section{Availability of data and materials}

Not applicable

\section{Competing interests}

The author declares that they have no competing interests

\section{Funding}

There was no specific funding for this report

\section{Author's contributions}

TL conceived, developed and wrote this article, approved the final version, and accepts full responsibility for its contents.

\section{Acknowledgements}

Not applicable 


\section{References}

1. Altman DG, Moher D. Declaration of transparency for each research article. BMJ. 2013;347:f4796.

2. Kasenda B, von Elm E, You J, Blumle A, Tomonaga Y, Saccilotto R, et al. Prevalence, characteristics, and publication of discontinued randomized trials. JAMA. 2014;311:1045-51.

3. Akl EA, Briel M, You JJ, Sun X, Johnston BC, Busse JW, et al. Potential impact on estimated treatment effects of information lost to follow-up in randomised controlled trials (LOST-IT): systematic review. BMJ. 2012;344:e2809.

4. Pocock SJ, Stone GW. The primary outcome is positive - is that good enough? N Engl J Med. 2016;375:971-9.

5. Loken E, Gelman A. Measurement error and the replication crisis. Science. 2017;355:584-5.

6. Lang TA, Gould J, von Seidlein L, Lusingu JP, Mshamu S, Ismael S, et al. Approaching the community about screening children for a multicentre malaria vaccine trial. Int Health. 2012;4:47-54.

7. Divala TH, Mungwira RG, Laufer MK. Moving targets: the challenges of studying infectious diseases among pregnant women in resource limited settings. Vaccine. 2015;33:6401-5.

8. Negussie H, Addissie T, Addissie A, Davey G. Preparing for and executing a randomised controlled trial of podoconiosis treatment in northern Ethiopia: the utility of rapid ethical assessment. PLoS Negl Trop Dis. 2016;10:e0004531.

9. Participants in the Community Engagement and Consent Workshop, Kilifi, Kenya, March 2011. Consent and community engagement in diverse research contexts. J Empir Res Hum Res Ethics. 2013;8:1-18.

10. Health Research Authority. Defining research table. 2017. http://www.hradecisiontools.org.uk/research/docs/DefiningResearchTable_Oct2017-1.pdf. Accessed 22 Nov 2018.

11. World Medical Association. World Medical Association Declaration of Helsinki: ethical principles for medical research involving human subjects. JAMA. 2013;310:2191-4.

12. Maitland K, Molyneux S, Boga M, Kiguli S, Lang T. Use of deferred consent for severely ill children in a multi-centre phase III trial. Trials. 2011;12:90.

13. Gobat NH, Gal M, Francis NA, Hood K, Watkins A, Turner J, et al. Key stakeholder perceptions about consent to participate in acute illness research: a rapid, systematic review to inform epi/pandemic research preparedness. Trials. 2015;16:591.

14. Furyk J, McBain-Rigg K, Watt K, Emeto TI, Franklin RC, Franklin D, et al: Qualitative evaluation of a deferred consent process in paediatric emergency research: a PREDICT study. BMJ Open. 2017;7:e018562.

15. RTS,S Clinical Trials Partnership. Efficacy and safety of RTS,S/AS01 malaria vaccine with or without a booster dose in infants and children in Africa: final results of a phase 3 , individually randomised, controlled trial. Lancet. 2015;386:31-45.

16. RTS,S Clinical Trials Partnership, Agnandji ST, Lell B, Fernandes JF, Abossolo BP, Methogo BG, et al. A phase 3 trial of RTS,S/AS01 malaria vaccine in African infants. N Engl J Med. 2012;367:2284-95. 
17. RTS,S Clinical Trials Partnership, Agnandji ST, Lell B, Soulanoudjingar SS, Fernandes JF, Abossolo BP, et al. First results of phase 3 trial of RTS,S/AS01 malaria vaccine in African children. N Engl J Med. 2011;365:1863-75.

18. Lentz J, Kennett M, Perlmutter J, Forrest A. Paving the way to a more effective informed consent process: recommendations from the Clinical Trials Transformation Initiative. Contemp Clin Trials. 2016;49:65-9.

19. Negussie H, Kassahun MM, Fegan G, Njuguna P, Enquselassie F, McKay A, et al. Podoconiosis treatment in northern Ethiopia (GoLBet): study protocol for a randomised controlled trial. Trials. 2015;16:307.

20. Deribe K, Brooker SJ, Pullan RL, Sime H, Gebretsadik A, Assefa A, et al. Epidemiology and individual, household and geographical risk factors of podoconiosis in Ethiopia: results from the first nationwide mapping. Am J Trop Med Hyg. 2015;92:148-58.

21. Mousley E, Deribe K, Tamiru A, Davey G. The impact of podoconiosis on quality of life in northern Ethiopia. Health Qual Life Outcomes. 2013;11:122.

22. Henok L, Davey, G. Validation of the Dermatology Life Quality Index among patients with podoconiosis in southern Ethiopia. British Journal of Dermatology. 2008;159:903-6.

23. Sikorski C, Ashine M, Zeleke Z, Davey G. Effectiveness of a simple lymphoedema treatment regimen in podoconiosis management in southern Ethiopia: one year follow-up. PLoS Negl Trop Dis. 2010;4:e902.

24. Lang T. Ebola: embed research in outbreak response. Nature. 2015;524:29-31.

25. Dunning J, Kennedy SB, Antierens A, Whitehead J, Ciglenecki I, Carson G, et al. Experimental treatment of Ebola virus disease with brincidofovir. PLoS One. 2016;11:e0162199.

\section{Figures}

\section{Figure 1}

Summary of potential topics for discussion at the study walk-through meeting

\section{Figure 2}

An example pre-study process plan.

This figure has been included for illustrative purposes only and is not intended to be fully legible. 


\section{Figure 3}

An example post-initiation study process map.

This figure has been included for illustrative purposes only and is not intended to be fully legible.

\section{Figure 4}

A study walk-through output used to guide protocol development during the Ebola outbreak.

This figure has been included for illustrative purposes only and is not intended to be fully legible. 